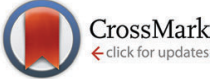

Cite this: Phys. Chem. Chem. Phys., 2016, 18, 14941

Received 26th February 2016, Accepted 6th May 2016

DOI: $10.1039 / c 6 c p 01335 j$

www.rsc.org/pccp

\title{
A comprehensive picture of the ultrafast excited-state dynamics of retinal $\dagger$
}

\author{
Oliver Flender, Mirko Scholz, Jonas Hölzer, Kawon Oum* and Thomas Lenzer*
}

\begin{abstract}
All-trans retinal is the chromophore of microbial rhodopsins initiating energy conversion and cellular signalling by subpicosecond photoinduced switching. Here, we provide detailed UV-Vis transient absorption experiments to disentangle the complex photochemistry of this polyene, which is governed by its terminal aldehyde group. After photoexcitation to the $\mathrm{S}_{2}\left({ }^{1} \mathrm{~B}_{\mathrm{u}}{ }^{+}\right)$state, the system exhibits polaritydependent branching, populating separate $\mathrm{S}_{1}\left({ }^{1} \mathrm{~A}_{\mathrm{g}}{ }^{-}\right)$and intramolecular charge transfer (ICT) species. In all solvents, population of a singlet $n \pi^{\star}$ state from $S_{1}$ is observed which represents the precursor of the $T_{1}$ triplet state. While triplet formation dominates in nonpolar solvents (67\% quantum yield), it is dramatically reduced in polar solvents $(4 \%)$. The channel closes completely upon replacing the aldehyde by a carboxyl group, due to an energetic up-shift of ${ }^{1} n \pi^{\star}$. In that case, internal conversion via the ICT species becomes the main pathway, with preferential formation of the initially excited isomer.
\end{abstract}

\section{Introduction}

Derivatives of retinal play a key role as chromophores in rhodopsin photoreceptor proteins and fulfil diverse functions, such as energy conversion, cellular signalling, vision and photopigment regeneration. ${ }^{1-3}$ In microbial rhodopsins, all-trans retinal (= all-trans $\beta$-apo-15-carotenal, compound 1 in Fig. S1, ESI $\dagger$ ) is connected as a protonated Schiff base (PSB) to an opsin apoprotein. Upon photoexcitation, the all-trans PSB undergoes excited-state photoisomerisation to the 13-cis isomer (compound 2 in Fig. S1, ESI $\dagger$ ). Characterising light-triggered processes of retinyl chromophores and the influence of the environment on their dynamics is therefore of prime importance to understand their function in vivo.

In the simplest model, the photoinduced dynamics of carotenoids involve three singlet electronic states: the $\mathrm{S}_{0}\left({ }^{1} \mathrm{~A}_{\mathrm{g}}{ }^{-}\right)$ ground state, the one-photon-forbidden $\mathrm{S}_{1}\left({ }^{1} \mathrm{~A}_{\mathrm{g}}{ }^{-}\right)$first excited

Universität Siegen, Physikalische Chemie, Adolf-Reichwein-Str. 2, 57076 Siegen, Germany.E-mail: oum@chemie.uni-siegen.de, lenzer@chemie.uni-siegen.de $\dagger$ Electronic supplementary information (ESI) available: Chemical structures; pump fluence dependence for transient absorption signals of all-trans retinal in $n$-hexane and acetonitrile; subtraction of the solvent contribution in the broadband transient absorption spectra; steady-state absorption spectra of all-trans and 13-cis retinal, their characteristic parameters and solvatochromic analysis; UV-Vis transient broadband absorption spectra of all-trans retinal; overview of time constants and quantum yields from the global analysis; species-associated spectra for all-trans retinal in different solvents; spectral fits and kinetics for all-trans retinal in different solvents; comparison with fit results from other kinetic models; steady-state absorption spectra for isomers of retinoic acid; UV-Vis broadband transient absorption spectra of 13-cis retinal; results of DFT/ TDDFT calculations for all-trans retinal and all-trans retinoic acid. See DOI: 10.1039/с6ср01335j state, and the optically "bright" $\mathrm{S}_{2}\left({ }^{1} \mathrm{~B}_{\mathrm{u}}{ }^{+}\right)$second excited state absorbing in the blue-green spectral region. In apocarotenals, the conjugated terminal aldehyde group alters the electronic properties drastically, especially those of the $S_{1}$ state. For instance, systems such as $\beta$-apo-12'-carotenal, show a strong decrease of the excited-state lifetime with increasing solvent polarity, in contrast to nonpolar carotenoids. ${ }^{4-7}$ This is ascribed to the formation of considerable intramolecular charge transfer (ICT) character in the $\mathrm{S}_{1}$ state. $^{8-10}$

Earlier studies suggest that the photochemistry of retinal is more complex. For instance, Yamaguchi and Hamaguchi performed ultrafast transient absorption spectroscopy of all-trans retinal in $n$-hexane and evaluated their data based on a kinetic model involving branching in the $S_{1}$ state: ${ }^{11}$ after $S_{2} \rightarrow S_{1}$ internal conversion (IC), $\mathrm{S}_{1}$ is depopulated via the " ${ }^{1} \mathrm{n} \pi^{*} /$ triplet channel" $\mathrm{S}_{1} \rightarrow{ }^{1} \mathrm{n} \pi^{*} \rightarrow \mathrm{T}_{1} \rightarrow \mathrm{S}_{0}$, forming exclusively the all-trans isomer ${ }^{12}$ with $74 \%$ quantum yield, and via the "photoisomerisation/IC channel" $\mathrm{S}_{1} \rightarrow p^{*} \rightarrow p \rightarrow \mathrm{S}_{0}$ involving perpendicular $\left(90^{\circ}\right)$ excited-state and ground state configurations $p^{*}$ and $p$, which lead to formation of trans and cis isomers. In a follow-up study, they reported transient absorption spectra in $n$-butanol, ${ }^{13}$ where the lifetime of the $S_{1}$ state was $1.6 \mathrm{ps}$, and thus slower than in $n$-hexane (0.56 ps). In $n$-butanol, stimulated emission (SE) was observed above $620 \mathrm{~nm}$, in agreement with SE kinetics previously detected in other polar solvents. ${ }^{14,15}$ Later on, Polívka et al. ${ }^{7}$ ascribed this red emission to an ICT state, based on an analogous assignment of SE features observed for $\beta$-apo-12' -carotenal, $\beta$-apo- $8^{\prime}$-carotenal ${ }^{5-7}$ and $\beta$-apo- $12^{\prime}$-carotenoic acid. ${ }^{16}$

Although these experiments have improved the understanding of retinal photochemistry, important pieces of information are still missing, such as (a) the complete spectral characterisation 
and ultrafast dynamics of the ${ }^{1} \mathrm{n} \pi^{*}$ state in different solvents, (b) the determination of solvent-dependent lifetimes and quantum yields for the processes in the ${ }^{1} \mathrm{n} \pi^{*}$ /triplet and photoisomerisation/ IC channels, (c) the possible influence of ICT character in the excited electronic state manifold, and (d) an explanation for the fast excited-state decay of retinal which is a priori unexpected for such a short-chain polyene species. We will address all of these points and arrive at a comprehensive description of the excited-state dynamics of all-trans retinal, with the aid of complementary experiments for the closely related all-trans retinoic acid (compound 3 in Fig. S1, ESI $\dagger$ ) and 13-cis retinal.

\section{Experimental}

\subsection{Substances}

All-trans retinal and all-trans retinoic acid were kindly provided in double-recrystallized form by BASF SE (Prof. Bernd Schäfer), 13-cis retinal was purchased from Toronto Research Chemicals and used without further purification. Purities were $>97 \%$ for the all-trans retinoids, 95\% for 13-cis retinal, and 99\% or better for the organic solvents.

\subsection{Pump - supercontinuum probe (PSCP) spectroscopy}

Ultrafast broadband transient absorption experiments were carried out using UV-Vis pump - supercontinuum probe spectroscopy at $920 \mathrm{~Hz}$ repetition frequency. ${ }^{17-19}$ All-trans retinal was excited by $400 \mathrm{~nm}$ pulses at every second laser shot (laser pulse fluence $c a .0 .23 \mathrm{~mJ} \mathrm{~cm}^{-2}$ ). Extended spectral coverage into the deeper UV region was achieved by employing a $400 \mathrm{~nm}$-seeded multifilament supercontinuum generated in a $2 \mathrm{~mm}$ thick translating $\mathrm{CaF}_{2}$ plate as probe pulse (260-660 nm, beam diameter $c a .130 \mu \mathrm{m})$. The relative pump-probe polarization was set at magic angle $\left(54.7^{\circ}\right)$. The pump-probe intensity crosscorrelation time varied in the range 57-84 fs in the different experiments, with a time zero accuracy of $c a .10 \mathrm{fs}$. To avoid any influence of photoinduced sample degradation, $10-15 \mathrm{~mL}$ of a nitrogen-saturated solution of all-trans retinal, 13-cis retinal or all-trans retinoic acid in the organic solvent of interest was passed through a flow cell (path length $1 \mathrm{~mm}$, window thickness $200 \mu \mathrm{m}$ ), so that the sample volume was exchanged after each pump-probe cycle. Solutions with an OD of 0.2 (at $400 \mathrm{~nm}$, $1 \mathrm{~mm}$ path length) were employed. Under these conditions, the transient absorption signals were in the linear regime, as confirmed in separate experiments (Fig. S2-S7, ESI $\dagger$ ). Steadystate absorption spectra of the sample solutions were recorded on a Varian Cary 5000 dual-beam spectrometer.

\subsection{Data processing and global kinetic analysis procedure}

The PSCP spectra were chirp-corrected by using the coherent response of the respective pure solvents. The coherent solvent signals were in all cases subtracted from the transient absorption data of retinal to obtain the pure chromophore dynamics (Fig. S8, ESI $\dagger$ ).

Each data set, consisting of 512 kinetic traces, was subjected to a global modelling procedure employing the kinetic scheme discussed later on. Species-associated spectra were parametrised by a sufficiently large number of Gaussian functions. During the modelling, the known, separately measured absolute $\mathrm{S}_{0}$ absorption spectra for the trans/cis isomers of retinal and retinoic acid were kept fixed. In addition, the following boundary conditions based on existing experimental data were imposed: total isomerisation quantum yields of $\Phi_{\text {cis }}=12 \%$ ( $n$-hexane, $n$-hexadecane) and $\Phi_{\text {cis }}=33 \%$ (ethanol), based on the measurements of Waddell et al. and Ganapathy and co-workers, ${ }^{20,21}$ were employed with an allowed variation of $\pm 15 \%$. Note that this only slightly influences the amplitude of the $\mathrm{T}_{1}$ triplet spectrum in the overlap region with the $S_{0}$ ground state bleach (GSB), with the $\mathrm{T}_{1}$ peak being unaffected. Next we assumed that all-trans retinal does not isomerise in the triplet channel, as found in independent transient FTIR experiments of Yuzawa and Hamaguchi. ${ }^{12}$ Finally, absolute peak absorption coefficients (all in units of $\mathrm{M}^{-1} \mathrm{~cm}^{-1}$ ) for the $\mathrm{T}_{1}$ triplet band are available from pulsed laser photolysis experiments of all-trans retinal in a few solvents, albeit partially with either unknown or substantial uncertainties: 70000 (n-hexane), ${ }^{22-25} 78600$ (THF), ${ }^{26} 68000$ (methanol) ${ }^{26,27}$ and 63000 (acetonitrile). ${ }^{28}$ During the fitting procedure, the peak value of the fitted $\mathrm{T}_{1}$ spectrum was forced to stay within $\pm 10 \%$ of these values.

The parameters, such as the position, width and height of the Gaussian functions describing the excited-state spectra and the rate constants, were optimised simultaneously to arrive at the best fit, which included convolution with the experimental time response, the latter one being determined from the pure solvent signal.

\subsection{DFT/TDDFT calculations}

Calculations for all-trans retinal and all-trans retinoic acid were performed based on the procedures described in our previous publications: ${ }^{29-31}$ the structure of the ground electronic state was optimized using DFT employing the B3LYP functional and a $6-31 G(d)$ basis set. The energies of the three lowest excited states (vertical excitation) were determined by TDDFT using SVWN, BLYP, B3LYP, MPW1K and CAM-B3LYP functionals and a $6-31+G(d)$ basis set.

\section{Results and discussion}

\subsection{Ultrafast dynamics of all-trans retinal and all-trans retinoic acid in different solvents}

UV-Vis steady-state absorption spectra of all-trans retinal and 13-cis retinal in various solvents are summarised in the ESI $\dagger$ (Fig. S9). The $\mathrm{S}_{0}\left({ }^{1} \mathrm{~A}_{\mathrm{g}}{ }^{-}\right) \rightarrow \mathrm{S}_{2}\left({ }^{1} \mathrm{~B}_{\mathrm{u}}{ }^{+}\right)$band maximum is located slightly below $400 \mathrm{~nm}$. The spectrum of the all-trans species has a larger absorption coefficient and red-shift than 13-cis. A discussion of the solvent-dependence of the absorption spectra is included in the ESI $\dagger$ (Fig. S9-S13), with the main finding that several factors, such as increasing solvent polarisability, polarity and hydrogen-bonding capability lead to an increased spectral red-shift. 
The $400 \mathrm{~nm}$ pump pulse used in the time-resolved experiments excites predominantly the $\mathrm{S}_{0} \rightarrow \mathrm{S}_{2}$ transition, with the weakly absorbing $\mathrm{S}_{1}\left({ }^{1} \mathrm{~A}_{\mathrm{g}}{ }^{-}\right)$state estimated to lie $2300 \mathrm{~cm}^{-1}$ below $\mathrm{S}_{2} \cdot{ }^{32}$ Fig. 1 shows transient UV-Vis PSCP broadband absorption spectra of all-trans retinal and all-trans retinoic acid in $n$-hexane. Fig. 2 compares the solvent dependence of the PSCP spectra of all-trans retinal in $n$-hexadecane, diisopropyl ether, THF and methanol as contour plots (corresponding transient spectra are included in the ESI, $\dagger$ Fig. S14-S19). The initially populated $\mathrm{S}_{2}$ state shows pronounced excited-state absorption (ESA) with a peak at $600 \mathrm{~nm}$ accompanied by an $\mathrm{S}_{0} \rightarrow \mathrm{S}_{2}$ GSB centred below $400 \mathrm{~nm}$. Four central aspects are immediately clear upon inspection of the subsequent spectral development:

(a) For retinal in $n$-hexane, there is the emergence of a sharp absorption band centred at $396 \mathrm{~nm}$ (Fig. 1(A), second panel, and (C)). It is assigned to ESA of the ${ }^{1} n \pi^{*}$ singlet state formed after intermediate population of the $S_{1}$ state (see below). The ${ }^{1} \mathrm{n} \pi^{*}$ state represents the precursor of the $T_{1}$ triplet state, and the latter one is formed at later times with a characteristic band peaking at $443 \mathrm{~nm},{ }^{26}$ see Fig. 1(A), bottom panel, and (C).

(b) The ${ }^{1} n \pi^{*} / T_{1}$ channel is closing down completely upon replacement of the terminal aldehyde group (retinal) by a carboxyl function (retinoic acid), see Fig. 1(B), second panel. There are no spectral fingerprints of the ${ }^{1} n \pi^{*}$ and $T_{1}$ states.

(c) Spectral features of the ${ }^{1} n \pi^{*}$ state of all-trans retinal become weaker with increasing solvent polarity, see Fig. 2(A-D) and Fig. S14-S19 (ESI $\dagger$ ). This suggests a strong reduction of the quantum yield for ${ }^{1} n \pi^{*}$ and $T_{1}$ formation. Still, in methanol and acetonitrile, weak ${ }^{1} n \pi^{*}$ and well-visible $T_{1}$ spectral features are present. A comparison of the solvent-dependent band shapes is shown in Fig. 3. In (A), at 0.17 ps the ${ }^{1} n \pi^{*}$ ESA band shows a sharp peak at $396 \mathrm{~nm}$ from nonpolar solvents up to diisopropyl ether. The band becomes much weaker in more polar solvents and also develops at later time (Fig. S16-S19, ESI $\dagger$ ), already suggesting delayed formation of ${ }^{1} \mathrm{n} \pi^{*}$ which is confirmed by the global analysis below. In (B), the long-lived $\mathrm{T}_{1} \rightarrow \mathrm{T}_{n}$ absorption develops from a narrow band in $n$-hexadecane $/ n$-hexane into a broader band with a long-wavelength tail for polar solvents, such as methanol or acetonitrile.

(d) The ESA band of all-trans retinoic acid (Fig. 1(B), second panel) featuring two peaks at 470 and $550 \mathrm{~nm}$ arises from an $\mathrm{S}_{1}$ /ICT species, which is populated from $\mathrm{S}_{2}$, see below. A very similar ESA band is also observed for all-trans retinal, with peaks located at 480 and $540 \mathrm{~nm}$ (Fig. 1(A), second panel). The appearance of the $S_{1}$ and ICT bands of all-trans retinal changes drastically with increasing polarity: from a double-peak structure in nonpolar solvents to a pronounced single peak band with a blue shoulder and an SE feature to the red (Fig. 3(A)). Most importantly, the kinetics in this ESA band exhibit a biexponential decay (e.g. at $530 \mathrm{~nm}$ ), strongly suggesting that two distinguishable species contribute in this spectral region which decay independently via separate relaxation channels.
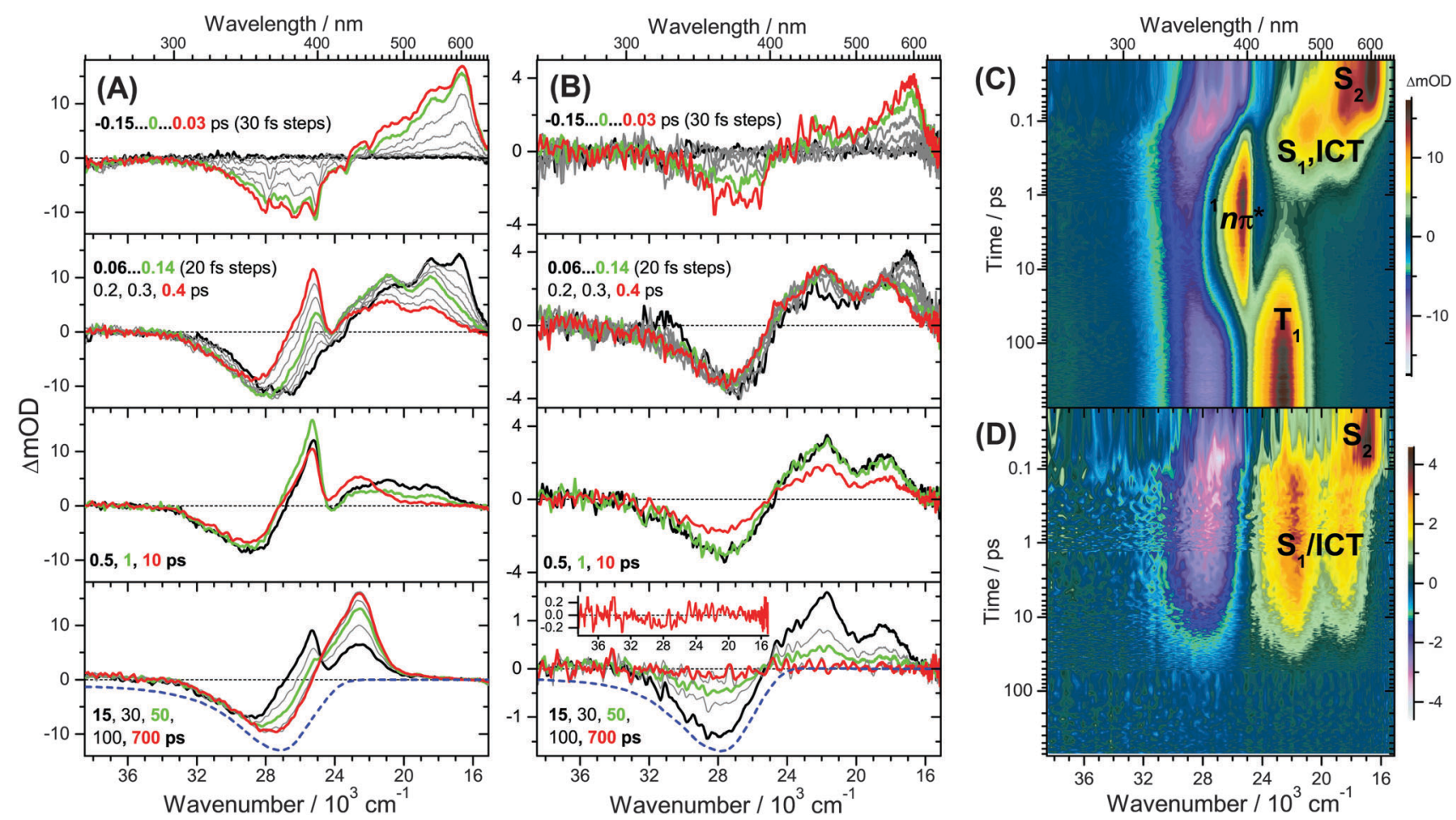

Fig. 1 Transient UV-Vis PSCP absorption spectra of all-trans retinal (A) and all-trans retinoic acid (B) in $n$-hexane upon excitation at $400 \mathrm{~nm}$. (Top panel) 0.15-0.03 ps with 30 fs steps; (2nd panel) 0.06-0.14 ps with 20 fs steps, and 0.2, 0.3 and 0.4 ps; (3rd panel) 0.5, 1 and 10 ps; (bottom panel) 15, 30, 50, 100 and 700 ps (inset in (B): magnified spectrum at 700 ps). Selected transient spectra are shown as thick coloured lines for guidance. The blue-dashed lines in the bottom panels are the scaled and inverted steady-state absorption spectra. The corresponding contour plots for all-trans retinal (C) and for all-trans retinoic acid (D) are presented with the symbols of the electronically excited species used in the global kinetic analysis. The concentration of retinoic acid was kept low $\left(3 \times 10^{-5} \mathrm{M}\right)$ to avoid any influence of dimer formation on the spectra. This leads to a reduced signal-to-noise ratio. 

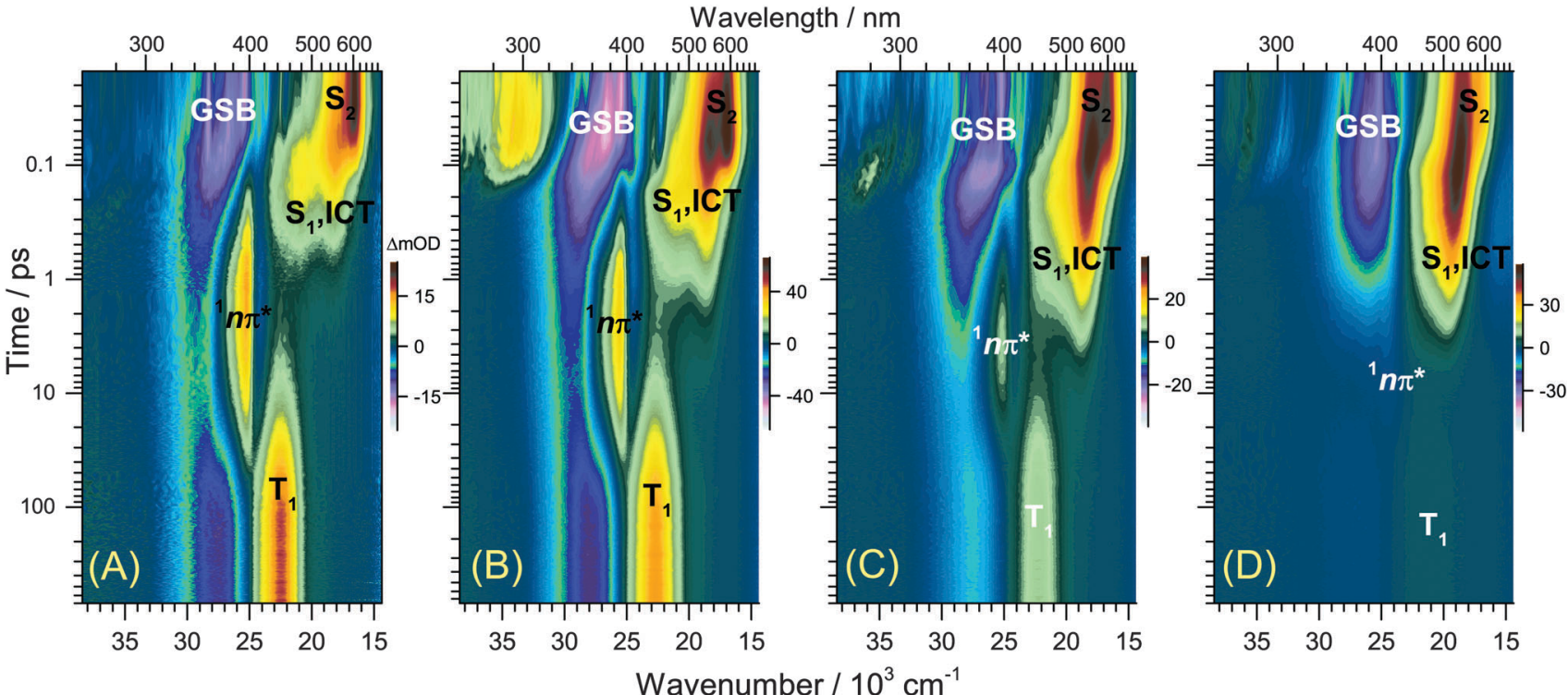

Fig. 2 Contour plots of transient UV-Vis PSCP absorption spectra of all-trans retinal in $n$-hexadecane (A), diisopropyl ether (B), THF (C) and methanol (D) upon $\mathrm{S}_{0} \rightarrow \mathrm{S}_{2}$ excitation at $400 \mathrm{~nm}$. Note the gradual disappearance of the ${ }^{1} \mathrm{n} \pi^{\star} /$ triplet channel with increasing solvent polarity. The absorption feature around $300 \mathrm{~nm}$ in (B) at short times (100 fs) is due to solvent contributions which cannot be removed perfectly by subtraction of the pure solvent spectrum.

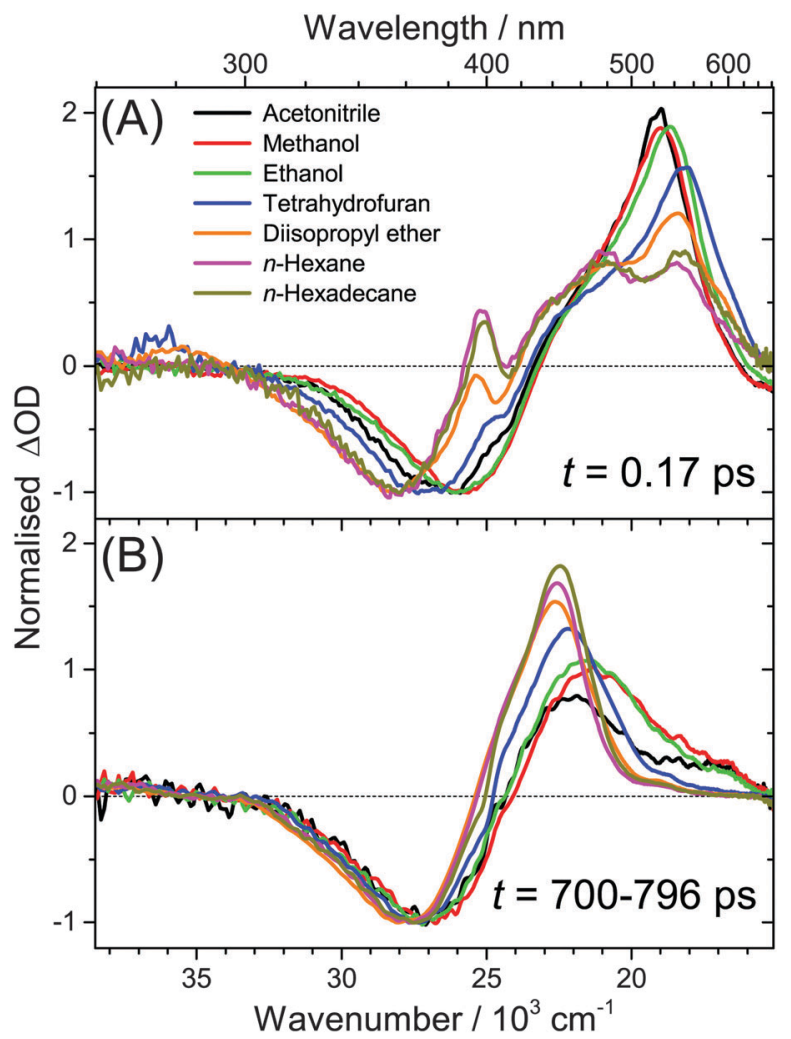

Fig. 3 Comparison of broadband transient absorption spectra of all-trans retinal in the organic solvents $n$-hexadecane, $n$-hexane, diisopropyl ether, THF, ethanol, methanol and acetonitrile. Spectra are normalised at the maximum GSB amplitude. (A) Spectra at $t=0.17$ ps: here, the spectral features of the $S_{1}$ and ICT species (above $400 \mathrm{~nm}$ ) and the rise of the ${ }^{1} n \pi^{*}$ absorption (around $396 \mathrm{~nm}$ ) are observed. (B) Spectra averaged over the time range 700-796 ps: the long-lived $T_{1} \rightarrow T_{n}$ triplet absorption band above $400 \mathrm{~nm}$ is clearly visible.

\subsection{Kinetic model}

Several kinetic schemes were explored to model the transient spectra of retinal. The best model reproducing all details of the dynamics is found in Fig. 4(A). As a central feature of the mechanism, separate $S_{1}$ and ICT species are populated from $S_{2}$ by IC. $\mathrm{S}_{1}$ and ICT decay independently to the ground electronic state, forming all-trans and cis isomers in $\mathrm{S}_{0}$. The $\mathrm{S}_{1}$ state is also the precursor of the ${ }^{1} n \pi^{*}$ state which forms the long-lived $T_{1}$ state (all-trans isomer) by ISC. The resulting time constants and quantum yields are summarized in Table S2, ESI. $\dagger$ Key parameters, such as the polarity dependence of quantum yields and selected time constants are highlighted in Fig. 4(B-E). Speciesassociated spectra (SAS) featuring absolute absorption coefficients are shown in Fig. 5. Additional SAS, species contributions to the spectra at selected times and kinetic traces including simulations are included in the ESI $\dagger$ (Fig. S20-S24).

We note that other kinetic schemes failed to reproduce central aspects of the spectral dynamics: for instance, a model employing branching from $\mathrm{S}_{2}$ forming the three species $\mathrm{S}_{1}$, ICT and ${ }^{1} \mathrm{n} \pi^{*}$ was abandoned, because it did not reproduce the delayed rise of the ${ }^{1} n \pi^{*}$ band, suggesting that $S_{1}$ must act as an intermediate for ${ }^{1} \mathrm{n} \pi^{*}$ formation, see ESI $\dagger$ (Fig. S25).

Furthermore, a model featuring a single $\mathrm{S}_{1} / \mathrm{ICT}$ state was tested for retinal. However, a single state should exhibit monoexponential decay behaviour. For instance, the build-up of population in the ${ }^{1} \mathrm{n} \pi^{*}$ state in nonpolar alkanes and THF $\left(\tau_{1, \mathrm{n} \pi^{*}} \leq 0.56 \mathrm{ps}\right.$ in Table S2, ESI $\left.\dagger\right)$ then would quickly drain population from $\mathrm{S}_{1} / \mathrm{ICT}$. It therefore immediately turned out that the second slower component observed in the spectral decay of the ESA band cannot be modelled, see Fig. S26, ESI. $\dagger$ Therefore separate $S_{1}$ and ICT species are clearly identified. 
(A)

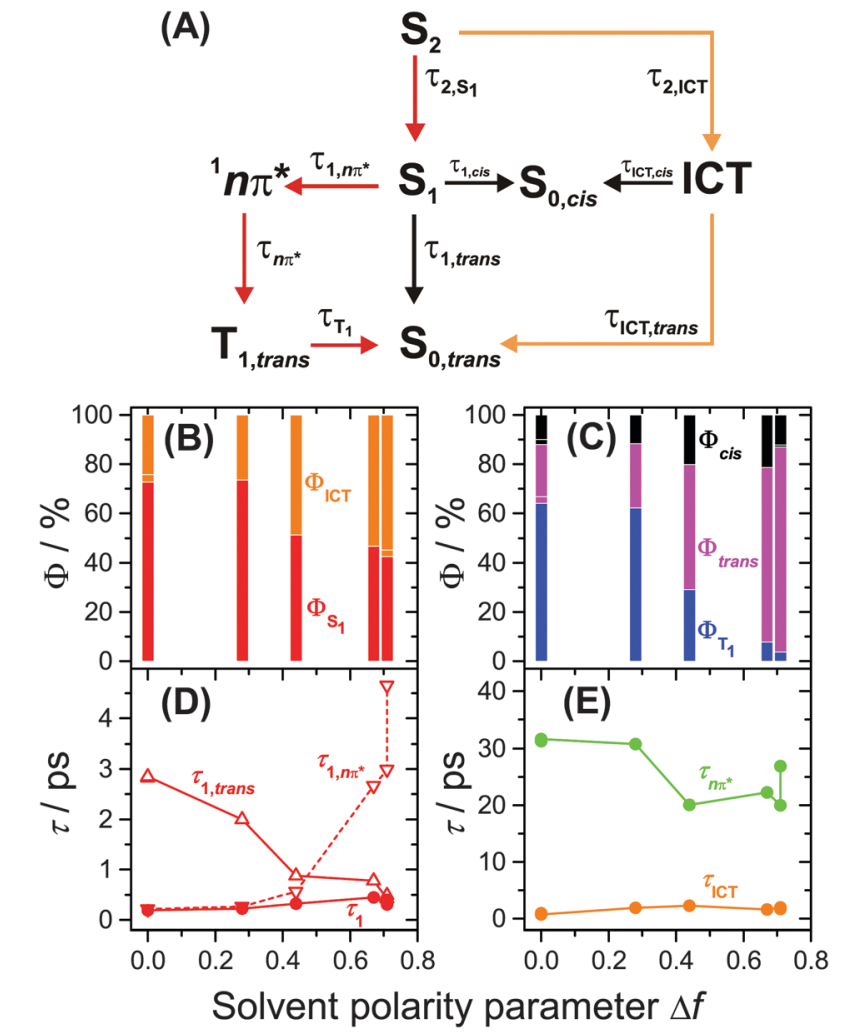

Fig. 4 Kinetic model for all-trans retinal photochemistry and key parameters of the global kinetic analysis. (A) Kinetic model: $S_{2}$ is initially populated from $S_{0, t r a n s}$ by photoexcitation. The 13 -cis isomer is the predominant cis isomerisation product, and according to the experiments of Yuzawa and Hamaguchi, ${ }^{12}$ the $T_{1}$ species has an all-trans configuration. The red pathway dominates in nonpolar solvents, whereas the orange one prevails in highly polar solvents. (B) Branching ratios from $S_{2}$ into $S_{1}$ (red) and ICT (orange). (C) Branching ratios for formation of $T_{1}$ (blue), $S_{0}$ all-trans (magenta) and $\mathrm{S}_{0, \text { cis }}$ (black). (D) Total lifetime $\tau_{1}$ of the $\mathrm{S}_{1}$ state and its contributions from the ${ }^{1} n \pi^{\star} / T_{1}$ channel $\left(\tau_{1, n \pi^{*}}\right)$ and internal conversion channel to $S_{0, \text { trans }}\left(\tau_{1, \text { trans }}\right)$. (E) Total lifetime of ICT (orange) and ${ }^{1} n \pi^{\star}$ (green).

The obvious question then arises if the $\mathrm{S}_{1}$ and ICT species might be in equilibrium. Based on our modelling we conclude that a "fast" equilibrium with forward and backward reactions on the picosecond timescale is not compatible with the experimental data, because the fastest channel dictates the decay of the ESA band, and it becomes impossible to model the slower decay component. Yet, we cannot exclude the presence of a slow equilibrium (with time constants of several picoseconds for forward and backward reaction). This implies the presence of an appreciable barrier on the potential energy surface between $S_{1}$ and ICT suppressing interconversion of the two species on the timescale of IC to the ground state. This further corroborates our final model in Fig. 4(A) assuming that the $\mathrm{S}_{1}$ and ICT species are effectively separated.

Lastly, the inclusion of trans-cis isomerisation in the mechanism of Fig. 4(A) is imperative for two reasons: the "persistent bleach" in the GSB region at $355 \mathrm{~nm}$ for all-trans retinoic acid in $n$-hexane in Fig. 1(B), bottom panel (in the absence of any triplet formation), directly proves the formation of cis-species exhibiting smaller absorption coefficients. We confirmed this

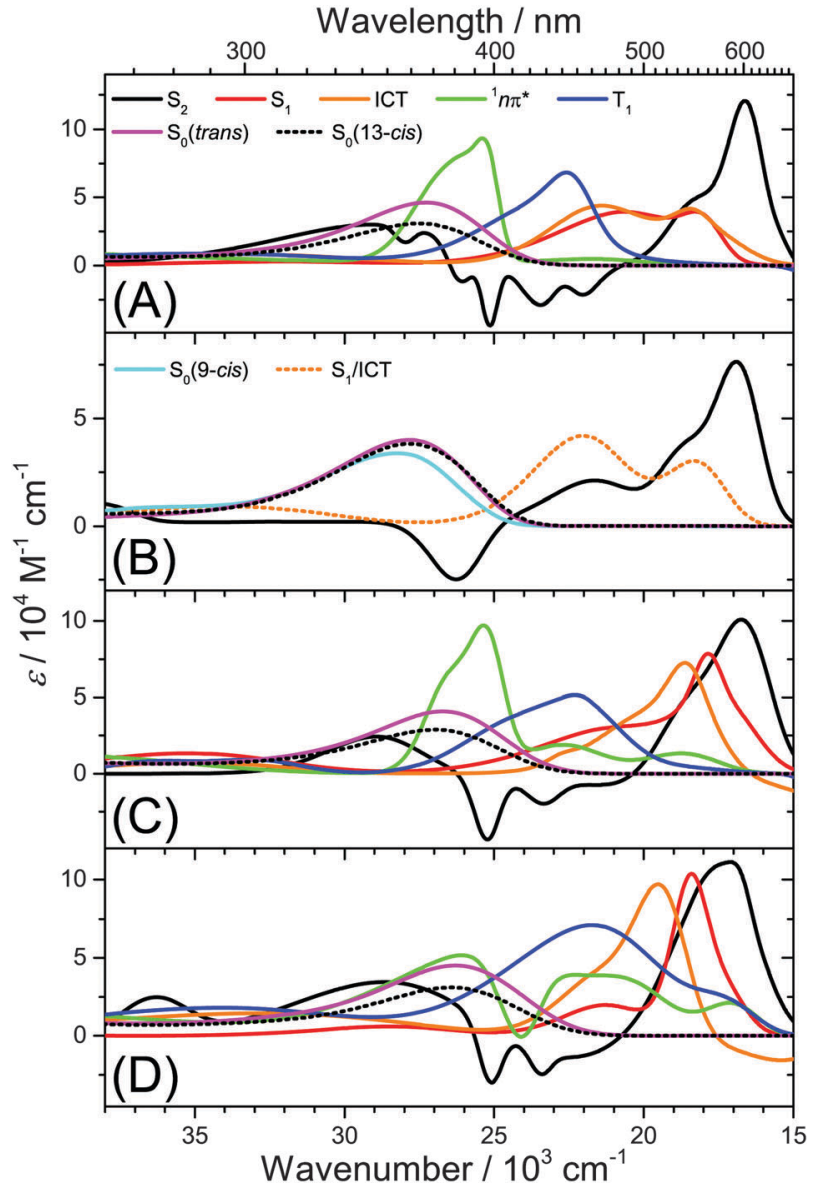

Fig. 5 Species-associated spectra from global kinetic analysis. (A) All-trans retinal in $n$-hexane, (B) all-trans retinoic acid in $n$-hexane, (C) all-trans retinal in THF, and (D) all-trans retinal in methanol.

by recording UV-Vis steady-state absorption spectra of different retinoic acid isomers (Fig. S27, ESI $\dagger$ ). For all-trans retinal, the situation is more complicated, because there are long-lived spectral contributions of the triplet band and its corresponding GSB contribution. Yet, earlier experiments employing HPLC analysis of primary photoproducts of all-trans retinal provided an estimate for the quantum yield of cis-isomer formation and confirmed that trans-cis isomerisation in the excited state indeed takes place, with 13-cis representing the dominant product. $^{20,21}$

\subsection{Polarity-dependent excited-state dynamics of all-trans retinal}

Having established the plausibility of the kinetic scheme in Fig. 4(A), we now turn toward the interpretation of the time constants and species-associated spectra obtained from the analysis. The lifetime of the $\mathrm{S}_{2}$ state is extremely short in all solvents, e.g. 55 and $60 \mathrm{fs}$ in $n$-hexane and $n$-hexadecane, respectively. This supports our previously observed trend that the $\mathrm{S}_{2}$ lifetime of apocarotenals systematically decreases upon shortening of the polyene chain. ${ }^{33}$ In all cases, the $\mathrm{S}_{2} \rightarrow \mathrm{S}_{n}$ spectrum is characterised by a sharp peak at ca. $600 \mathrm{~nm}$ with a shoulder extending to shorter wavelengths. 
We observe a systematic decrease of the quantum yield for $\mathrm{S}_{1}$ formation with solvent polarity. For instance, it is $73 \%$ in $n$-hexane and only $43 \%$ in acetonitrile, thus population of ICT is favoured in polar solvents (Fig. 4(B)). The latter finding is in agreement with the characteristic change of the experimental ESA band shape and the appearance of pronounced SE above $600 \mathrm{~nm}$ from diisopropyl ether onwards (Fig. 5(C and D), orange lines, and Fig. S20, ESI $\dagger$ ).

The species-associated spectra of $S_{1}$ (red) and ICT (orange) exhibit systematic changes with polarity: in nonpolar solvents ( $n$-hexane, $n$-hexadecane), the spectra contain two broad peaks and look similar. In polar solvents, the ICT spectra show a pronounced single peak with a shoulder toward shorter wavelengths and a distinct SE feature above $600 \mathrm{~nm}$. The $S_{1}$ spectra are more red-shifted and show no SE band. We therefore conclude that the $S_{1}$ species is a higher-energy local minimum of the first electronically excited state (assuming a similar energetic position of the upper state in the ESA transitions of $S_{1}$ and ICT). $S_{1}$ has access to the ${ }^{1} n \pi^{*}$ state and is separated from the lower-energy ICT part of the potential by an appreciable barrier. The charge-transfer character of ICT is less pronounced in nonpolar solvents due to the missing dipolar solvent stabilization. Therefore the SE feature is likely hidden beneath the stronger ESA band due to its much smaller Stokes shift in nonpolar solvents.

The formation of ${ }^{1} \mathrm{n} \pi^{*}$ from $\mathrm{S}_{1}$ significantly slows down with increasing solvent polarity, from $0.21 \mathrm{ps}$ in $n$-hexane to $3.0 \mathrm{ps}$ in acetonitrile and, at the same time, internal conversion to $\mathrm{S}_{0}$ accelerates (Fig. 4(D)). These two effects lead to a dramatic reduction of the triplet quantum yield from $64 \%$ in $n$-hexane to $4 \%$ in acetonitrile (Fig. $4(\mathrm{C})$ ). It is reasonable to assume that a polarity-induced shift of the ${ }^{1} n \pi^{*}, S_{1}$ and ICT energy levels is responsible for this effect. Most importantly, an increase of polarity tends to increase the energy of ${ }^{1} n \pi^{*}$ species relative to $\mathrm{S}_{1}$ and ICT. ${ }^{34}$ This likely reduces the coupling between $\mathrm{S}_{1}$ and ${ }^{1} \mathrm{n} \pi^{*}$ and slows down the IC rate for ${ }^{1} \mathrm{n} \pi^{*}$ formation, favouring IC to $S_{0}$ instead.

We completely characterise the ${ }^{1} n \pi^{*}$ state spectra in alkanes and diisopropyl ether (Fig. 5, green lines). The band is centred at $396 \mathrm{~nm}$ with a shoulder toward shorter wavelengths. In polar solvents, the shape of the ${ }^{1} n \pi^{*}$ spectrum should be taken with some caution, because its ESA band is weak (Fig. S17-S19, ESI $\dagger$ ), due to the small quantum yield for ${ }^{1} \mathrm{n} \pi^{*}$ formation and its subsequent decay to $\mathrm{T}_{1}$. Still, it is clearly visible that the ${ }^{1} n \pi^{*}$ band is located at about the same position as in nonpolar solvents and broadens.

The time constant for $T_{1}$ formation from ${ }^{1} n \pi^{*}$ in alkanes and diisopropyl ether is $c a .31 \mathrm{ps}$. This ISC process accelerates somewhat with increasing solvent polarity (Fig. 4(E)). The $T_{1}$ spectra in alkanes and diisopropyl ether are characterised by a main peak at ca. $443 \mathrm{~nm}$ (Fig. 5, blue lines, and Fig. 3), with a typical absorption coefficient of $c a .70000 \mathrm{M}^{-1} \mathrm{~cm}^{-1}$. In polar solvents, the $\mathrm{T}_{1}$ spectra broaden significantly.

\subsection{Influence of the type of terminal carbonyl group}

Fig. 1(B and D) demonstrated that the ${ }^{1} n \pi^{*} / T_{1}$ channel is completely shut down upon replacing the terminal aldehyde by a carboxyl group. Consequently, global modelling for all-trans retinoic acid in $n$-hexane is possible using the SAS shown in Fig. 5(B). We note that a single spectrum for $\mathrm{S}_{1} / \mathrm{ICT}$ suffices, because the whole ESA band decays monoexponentially. It has a shape which is similar to the $S_{1}$ and ICT species in all-trans retinal (Fig. 5(A)). The influence of the $\mathrm{COOH}$ group is twofold: it shifts the ${ }^{1} \mathrm{n} \pi^{*}$ state to higher energies, so that access from $S_{1}$ is no longer possible. Such an energetic upshift of ${ }^{1} n \pi^{*}$ states of carboxylic acids is known for shorter conjugated systems $^{35}$ and was also found in our DFT/TDDFT calculations reported in Section 3.6. Secondly, the ICT character is weaker for a $\mathrm{COOH}$ group than for a $\mathrm{CHO}$ group, as suggested by our previous studies of longer-conjugated $\beta$-apocarotenals and $\beta$-apocarotenoic acids. ${ }^{5,6,16,36}$ Therefore the excited-state ICT character of retinoic acid should be reduced and the barrier between the $S_{1}$ and ICT part should be small or even absent, leading to the monoexponential ESA decay. It also appears as if the larger energetic separation of the ${ }^{1} n \pi^{*}$ state from $S_{1} /$ ICT increases the IC lifetime for $\mathrm{S}_{1} / \mathrm{ICT} \rightarrow \mathrm{S}_{0}$ to $19 \mathrm{ps}$.

\subsection{Starting from the 13-cis isomer}

For further testing of our kinetic model for retinal excited-state dynamics, we carried out selected transient absorption experiments starting from 13-cis retinal. We focus on the limiting cases in nonpolar and polar solvents. Transient absorption spectra in $n$-hexane and acetonitrile are included in Fig. S28, ESI. $\dagger$ Results of the global kinetic modelling are found in Table S2, ESI. $\dagger$ At first glance, the transient spectra look similar to those of the all-trans isomer. Inspection of the global modelling results revealed however significant differences: the ${ }^{1} n \pi^{*} /$ triplet quantum yield is reduced, and 13-cis formation dominates. The latter becomes immediately clear upon inspection of the transient absorption spectrum of 13-cis retinal in $n$-hexane: it is shifted toward shorter wavelengths (Fig. S29, ESI $\dagger$ ). If a larger amount of all-trans isomer was formed, the triplet band should largely resemble the all-trans $\mathrm{T}_{1}$ band, which is obviously not the case. A similar argument holds for acetonitrile (Fig. S28(B), ESI $\dagger$ ): if an appreciable amount of all-trans isomer was formed, there should be no GSB at long times but a weak absorption feature, because of the higher absorption coefficient of the all-trans isomer (Fig. S9, ESI $\dagger$ ). This is also not the case. There is apparently a strong propensity for the recovery of the initially photoexcited isomer. We therefore conclude that the kinetic scheme in Fig. 4(A) is also applicable to the excited-state dynamics of 13-cis retinal, only the trans and cis denominations need to be exchanged.

\subsection{Comparison with DFT/TDDFT calculations}

We also carried out selected DFT/TDDFT calculations for all-trans retinal and all-trans retinoic acid. Although there are well-known limitations and drawbacks of this method when applied to polyene systems with charge-transfer character, ${ }^{37,38}$ valuable semi-quantitative information was extracted regarding the influence of the terminal carbonyl group. Transitions and oscillator strengths are summarised in Table S3 and Fig. S30 (ESI $\dagger$ ). 
Detachment and attachment electron densities ${ }^{39}$ for the three lowest excited singlet states are plotted in Fig. S31 (ESI $\dagger$ ).

We focus on the shifts of the electronic transitions upon replacement of the aldehyde by the carboxyl group. The optically dark $\mathrm{S}_{0} \rightarrow{ }^{1} \mathrm{n} \pi^{*}$ transition is clearly identified in the detachment/ attachment plots by the transfer of electron density from the oxygen lone pairs into the conjugated $\pi$-system (Fig. S31, ESI $\dagger$ ). For all functionals, the $\mathrm{S}_{0} \rightarrow{ }^{1} \mathrm{n} \pi^{*}$ transition is strongly shifted upward by 7000-10000 $\mathrm{cm}^{-1}$ (Table S3, Fig. S30, ESI $\dagger$ ). In contrast, the $\mathrm{S}_{0} \rightarrow{ }^{1} \mathrm{~A}_{\mathrm{g}}{ }^{-}$and $\mathrm{S}_{0} \rightarrow{ }^{1} \mathrm{~B}_{\mathrm{u}}{ }^{+} \pi \pi^{*}$ transitions exhibit only a mild up-shift of $450-800 \mathrm{~cm}^{-1}$. The calculations therefore provide a rationale for the switching-off of the ${ }^{1} n \pi^{*} /$ triplet channel observed in the time-resolved experiments upon replacing the $\mathrm{CHO}$ by the $\mathrm{COOH}$ group, because the ${ }^{1} n \pi^{*}$ state is then no longer accessible from ${ }^{1} \mathrm{~A}_{\mathrm{g}}{ }^{-}$. The calculations also confirm the slight blue-shift observed in the steady-state absorption spectra upon replacement of the aldehyde by the carboxyl group (Fig. 1(A) and (B), bottom panels).

A closer look however reveals substantial discrepancies between experiment and TDDFT calculations. For instance, in the case of all-trans retinal, ${ }^{1} \mathrm{n} \pi^{*}$ is no longer the lowest excited state for the functionals B3LYP, MPW1K and CAM-B3LYP. In addition, the spacing between the ${ }^{1} \mathrm{~B}_{\mathrm{u}}{ }^{+}$and the ${ }^{1} \mathrm{~A}_{\mathrm{g}}{ }^{-}$states and their oscillator strengths are not consistent with experiment. For reaching quantitative agreement, more accurate quantum chemical calculations of these systems in different solvents will therefore be needed. This would require the accurate determination of the energetic positions of the $S_{2}, S_{1}$, ICT and ${ }^{1} \mathrm{n} \pi^{*}$ species. However currently, such calculations are very difficult, because this would require the correct treatment of doubly excited configurations in these polyenes, ${ }^{37}$ a correct inclusion of the charge-transfer character introduced by the terminal carbonyl group, ${ }^{38}$ as well as an appropriate consideration of local solvent effects beyond a simple polarizable continuum approach, including solvent-dependent relaxation of the excited-state structures. Note also that, so far, even high-level gas-phase CASSCF and CASPT2 calculations for retinal strongly overestimate the energy of the bright $S_{0} \rightarrow S_{2}$ transition. ${ }^{40}$ Recent approaches, such as the one used by Frank, Birge and co-workers for the carbonyl carotenoid peridinin ${ }^{41}$ might be also valuable for a better understanding of the excited-state manifold of all-trans retinal and all-trans retinoic acid. In any case, we hope that the current experiments will serve as a useful benchmark for future high-level quantum chemical calculations of these systems.

\section{Conclusions}

The photochemistry of retinal is largely controlled by the ${ }^{1} \mathrm{n} \pi^{*}$ state. According to El-Sayed's rule, ${ }^{42-44}$ only an ${ }^{1} \mathrm{n} \pi^{*} \rightarrow \mathrm{T}_{1}$ ISC transition is sufficiently fast to efficiently compete with IC processes typically prevailing in carotenoids. We demonstrated that this channel is operative in all solvents and gradually closes with increasing solvent polarity. A complete suppression of the ${ }^{1} n \pi^{*}$ channel happens upon changing to a terminal substituent, such as carboxyl, which pushes up the energy of ${ }^{1} n \pi^{*}$.
Exactly this effect is also operative in the all-trans retinal Schiff base of microbial rhodopsins, as its ${ }^{1} n \pi^{*}$ state is known to be located at higher energy than for the corresponding aldehyde. ${ }^{45-50}$ Protonation of the Schiff base even completely removes its ability to form an ${ }^{1} \mathrm{n} \pi^{*}$ state. $^{51}$

In all solvents, isomerisation of the initially photoexcited species is inefficient, e.g. $<21 \%$ for all-trans retinal (Table S2, ESI $\dagger$ ). Such an intrinsically low quantum yield is unwanted for retinal's function as an efficient photoswitch. In microbial rhodopsins, nature has optimized it to $64 \%,{ }^{52,53}$ implying that the protein pocket promotes much more efficient trans-cis isomerisation. ${ }^{1}$

\section{Acknowledgements}

We acknowledge B. Schäfer from BASF SE for providing high purity all-trans retinal and all-trans retinoic acid samples for the current experiments. We are indebted to N. P. Ernsting (Humboldt University Berlin, Germany) and J. L. Pérez Lustres (University of Santiago de Compostela, Spain) as well as J. Troe, K. Luther, J. Schroeder, D. Schwarzer and A. M. Wodtke (Georg August University Göttingen, Germany) for their continuous support and advice.

\section{References}

1 O. P. Ernst, D. T. Lodowski, M. Elstner, P. Hegemann, L. S. Brown and H. Kandori, Chem. Rev., 2014, 114, 126.

2 A. Wand, I. Gdor, J. Zhu, M. Sheves and S. Ruhman, Annu. Rev. Phys. Chem., 2014, 64, 437.

3 P. D. Kiser, M. Golczak and K. Palczewski, Chem. Rev., 2014, 114, 194.

4 D. A. Wild, K. Winkler, S. Stalke, K. Oum and T. Lenzer, Phys. Chem. Chem. Phys., 2006, 8, 2499.

5 M. Kopczynski, F. Ehlers, T. Lenzer and K. Oum, J. Phys. Chem. A, 2007, 111, 5370.

6 K. Oum, P. W. Lohse, F. Ehlers, M. Scholz, M. Kopczynski and T. Lenzer, Angew. Chem., Int. Ed., 2010, 49, 2230.

7 T. Polívka, S. Kaligotla, P. Chábera and H. A. Frank, Phys. Chem. Chem. Phys., 2011, 13, 10787.

8 J. A. Bautista, R. E. Connors, B. B. Raju, R. G. Hiller, F. P. Sharples, D. Gosztola, M. R. Wasielewski and H. A. Frank, J. Phys. Chem. A, 1999, 103, 8751.

9 D. Zigmantas, T. Polívka, R. G. Hiller, A. Yartsev and V. Sundström, J. Phys. Chem. A, 2001, 105, 10296.

10 D. Zigmantas, R. G. Hiller, A. Yartsev, V. Sundström and T. Polívka, J. Phys. Chem. B, 2003, 107, 5339.

11 S. Yamaguchi and H. Hamaguchi, J. Chem. Phys., 1998, 109, 1397.

12 T. Yuzawa and H. Hamaguchi, J. Mol. Struct., 1995, 352/353, 489.

13 S. Yamaguchi and H. Hamaguchi, J. Phys. Chem. A, 2000, 104, 4272.

14 E. J. Larson, L. A. Friesen and C. K. Johnson, Chem. Phys. Lett., 1997, 265, 161. 
15 E. J. Larson, S. J. Pyszczynski and C. K. Johnson, J. Phys. Chem. A, 2001, 105, 8136.

16 S. Stalke, D. A. Wild, T. Lenzer, M. Kopczynski, P. W. Lohse and K. Oum, Phys. Chem. Chem. Phys., 2008, 10, 2180.

17 A. L. Dobryakov, S. A. Kovalenko, A. Weigel, J. L. Pérez Lustres, J. Lange, A. Müller and N. P. Ernsting, Rev. Sci. Instrum., 2010, 81, 113106.

18 K. Oum, T. Lenzer, M. Scholz, D. Y. Jung, O. Sul, B. J. Cho, J. Lange and A. Müller, J. Phys. Chem. C, 2014, 118, 6454.

19 L. F. Tietze, B. Waldecker, D. Ganapathy, C. Eichhorst, T. Lenzer, K. Oum, S. O. Reichmann and D. Stalke, Angew. Chem., Int. Ed., 2015, 54, 10317.

20 W. H. Waddell and K. Chihara, J. Am. Chem. Soc., 1981, 103, 7389.

21 S. Ganapathy and R. S. H. Liu, J. Am. Chem. Soc., 1992, 114, 3459.

22 B. Veyret, S. G. Davis, M. Yoshida and K. Weiss, J. Am. Chem. Soc., 1978, 100, 3283.

23 R. Bensasson, E. J. Land and T. G. Truscott, Photochem. Photobiol., 1973, 17, 53.

24 T. Rosenfeld, A. Alchalel and M. Ottolenghi, J. Phys. Chem., 1974, 78, 336.

25 R. Azerad, R. Bensasson, M. B. Cooper, E. A. Dawe and E. J. Land, presented on the Int. Conf. at the Calouste Gulbenkian Foundation Centre, Lisbon, Portugal, 1974, in: Excited States of Biological Molecules, ed. J. B. Birks, Wiley (London), 1976, p. 531.

26 W. Dawson and E. W. Abrahamson, J. Phys. Chem., 1962, 66, 2542.

27 M. M. Fisher and K. Weiss, Photochem. Photobiol., 1974, 20, 423.

28 P. K. Das and R. S. Becker, J. Am. Chem. Soc., 1979, $101,6348$.

29 K. Golibrzuch, F. Ehlers, M. Scholz, R. Oswald, T. Lenzer, K. Oum, H. Kim and S. Koo, Phys. Chem. Chem. Phys., 2011, 13, 6340.

30 K. Oum, P. W. Lohse, J. R. Klein, O. Flender, M. Scholz, A. Hagfeldt, G. Boschloo and T. Lenzer, Phys. Chem. Chem. Phys., 2013, 15, 3906.
31 K. Oum, O. Flender, P. W. Lohse, M. Scholz, A. Hagfeldt, G. Boschloo and T. Lenzer, Phys. Chem. Chem. Phys., 2014, 16, 8019.

32 R. R. Birge, J. A. Bennett, L. M. Hubbard, H. L. Fang, B. M. Pierce, D. S. Kliger and G. E. Leroi, J. Am. Chem. Soc., 1982, 104, 2919.

33 F. Ehlers, M. Scholz, J. Schimpfhauser, J. Bienert, K. Oum and T. Lenzer, Phys. Chem. Chem. Phys., 2015, 17, 10478.

34 T.-i. Lai, B. T. Lim and E. C. Lim, J. Am. Chem. Soc., 1982, 104, 7631.

35 J. D. Coyle, Chem. Rev., 1978, 78, 97.

36 P. W. Lohse, R. Bürsing, T. Lenzer and K. Oum, J. Phys. Chem. B, 2008, 112, 3048.

37 J. H. Starcke, M. Wormit, J. Schirmer and A. Dreuw, Chem. Phys., 2006, 329, 39.

38 A. Dreuw and M. Head-Gordon, Chem. Rev., 2005, 105, 4009.

39 M. Head-Gordon, A. M. Grana, D. Maurice and C. A. White, J. Phys. Chem., 1995, 99, 14261.

40 M. Merchán and R. González-Luque, J. Chem. Phys., 1997, 106, 1112.

41 N. L. Wagner, J. A. Greco, M. M. Enriquez, H. A. Frank and R. R. Birge, Biophys. J., 2013, 104, 1314.

42 M. A. El-Sayed, J. Chem. Phys., 1962, 36, 573.

43 M. A. El-Sayed, J. Chem. Phys., 1963, 38, 2834.

44 M. A. El-Sayed, J. Chem. Phys., 1964, 41, 2462.

45 K.-H. Grellmann, R. Memming and R. Livingston, J. Am. Chem. Soc., 1962, 84, 546.

46 W. H. Waddell, A. M. Schaffer and R. S. Becker, J. Am. Chem. Soc., 1973, 95, 8223.

47 R. S. Becker, G. Hug, P. K. Das, A. M. Schaffer, T. Takemura, N. Yamamoto and W. Waddell, J. Phys. Chem., 1976, 80, 2265.

48 R. S. Becker, P. K. Das and G. Kogan, Chem. Phys. Lett., 1979, 67, 463.

49 P. K. Das, G. Kogan and R. S. Becker, Photochem. Photobiol., 1979, 30, 689.

50 R. S. Becker, Photochem. Photobiol., 1988, 48, 369.

51 E. W. Abrahamson, R. G. Adams and V. J. Wulff, J. Phys. Chem., 1959, 63, 441.

52 R. Govindjee, S. P. Balashov and T. G. Ebrey, Biophys. J., 1990, 58, 597.

53 J. Tittor and D. Oesterhelt, FEBS Lett., 1990, 263, 269. 